\section{The materials making potassium-ion batteries possible}

You are probably familiar with
lithium-ion batteries that can
be found everywhere from
inside our mobile phones to
electric cars. However, lithium's
larger brother potassium
may soon find its way into
the batteries that power our
everyday lives. Dr Titus Masese
at the National Institute of
Advanced Industrial Science
and Technology in Osaka,
Japan, has been developing
new materials for electrodes
to help overcome some of the
current limitations of potassium-
ion battery technologies to
allow them to reach their
potential as a promising low-cost
rechargeable battery material
for energy storage.

nelectrical battery is any device One of the most famous types of that stores energy that can be rechargeable batteries is the lithium-ion A basic battery provides electricity, or batteries try to overcome his by a flow of electrons, by having a positively ande circh

When a battery is connected to an electrical circuit, chemical reactions within the battery cause electrons to start building up at the anode. Eventually, this pile-up of electrons becomes unstable and will move through the electrolyte separating the anode and cathode, and flow around the circuit, providing the necessary electrical power.

However, each of these chemical reactions depletes the stored potential energy in the battery. Rechargeable reversing the oxidation and reduction occur at the cathode and anode and this time, convert electrical energy to chemical energy. batty, where its high-power density . However, lithe ithe smanthones. not the only option for rechargeable battery technologies.

Dr Titus Masese at the National Institute of Advanced Industrial Science and Technology in Osaka, Japan has been working on new, potassium-based materials for developing potassiumion based rechargeable batteries. There are good motivations for doing this. Developments in higher-energy, longer-lifetime and lower-cost battery technologies are a key part of the necessary energy storage strategy required for a more sustainable future offer a lower-cost alteries may por a lower-cost alternative, partly as potassium is over eight hundred times

\section{A MATERIALS CHALLENGE} Part of the reason that phones today is some of the technical challenges with their development that people like Dr Masese and his colleagues are working to overcome. The name of from the type of chemical element.

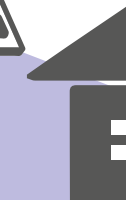

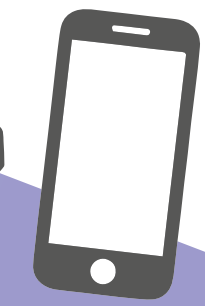

that is embedded in the electrodes. This ion, which is oppositely charged to the electrons produced in the battery, is released from the electrodes and moves The movorient for the battery performance as the movement of the electrons if the ion fats to move, the battery can discharge and fail to provide energy.

What Dr Masese and his colleagues have been successfully able to do is create honeycomb layered cathode frameworks that incorporate potassium ions and are capable of sustaining very high voltages. One of the challenges for using potassium ions in rechargeable batteries is that their large sizes can make it difficult to incorporate them in the tight-packed lattice frameworks that make up the type of electrons used in litium ion batteries. In anideal batery, he ions would be fast to release from the frans an, move reincorporated back into the frameworks as required.

The honeycomb structures that Dr Masese and his colleagues have pioneered it could potentially show the highest energy delivery as part of a battery. He has also been working to incorporate these into rechargeable potassium-ion batteries as a step towards more practica potassium-ion battery technologies.

\section{DURABLE ELECTRODES}

The secret of the success of Dr Masese's honeycomb structures lies in their ion recombination, a significant challenge

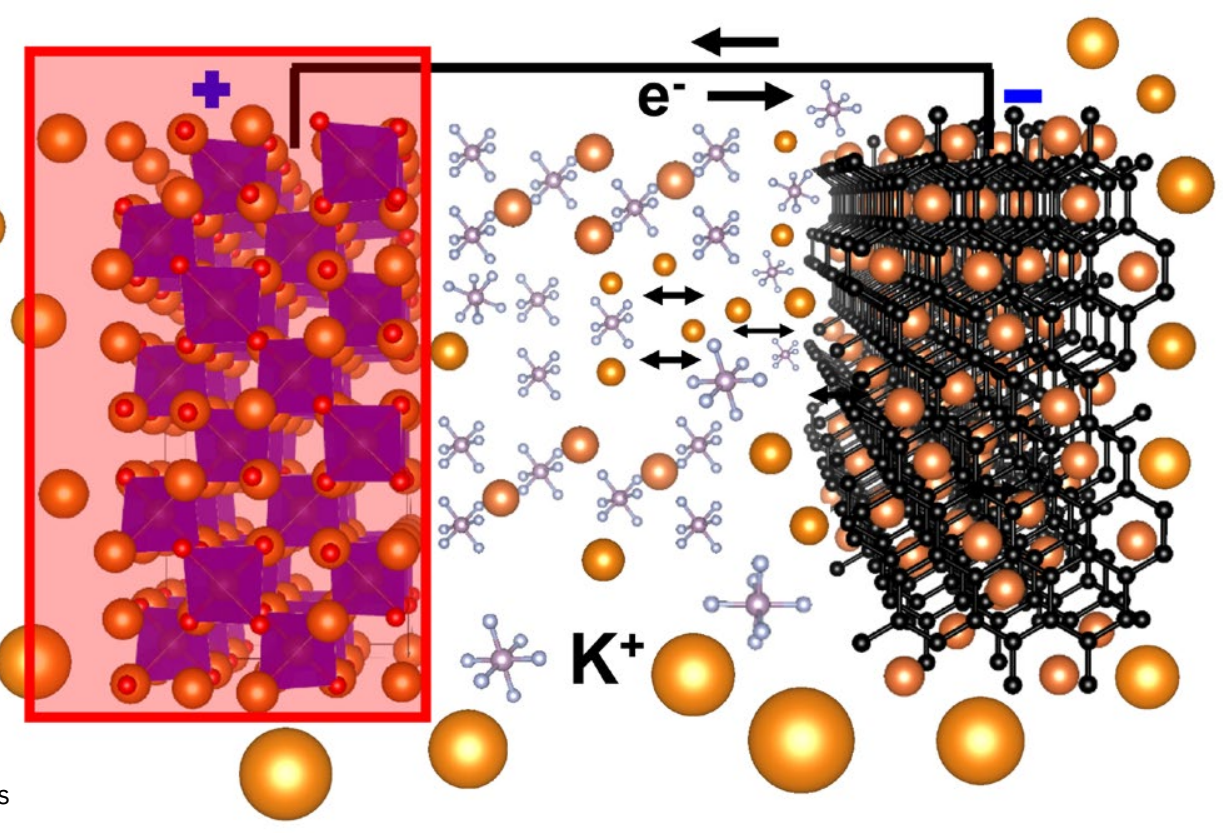

The honeycomb structures that Dr Masese has pioneered currently show the largest voltage for any layered cathode material.

due to the large size of the potassium potential lifetimes of potassium-ion ions. Many electrode materials are made batteries based on these cathodes. from highly organised, regular crystalline It is not just the cathode though that ion will remain in the same place. durability. As the electrolyte can also play However, recombination processes and a role in detrimental chemical reactions, unwanted chemical reactions between this too must be as benign as possible.

\section{damaging and aging of the battery. This FUTURE OF ELECTROLYTES} is why many phones undergo significant Dr Masese and his colleagues have been within fow ors of merformance even using ionic liquids as the electroly a few years of manufacture.

These honeycomb materials have shown to be high voltages the and maintain producing, which bodes well for the potassium in cels, or ellurafebased desirable. lonic liquids are unusual their behaviour as they are liquids that contain dissolved salts that

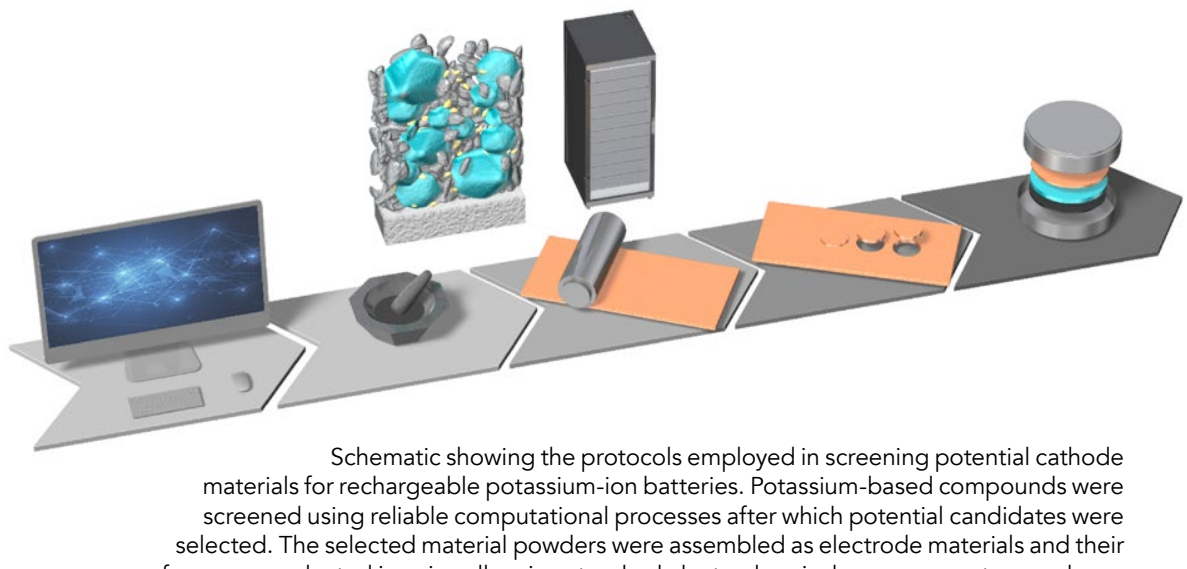




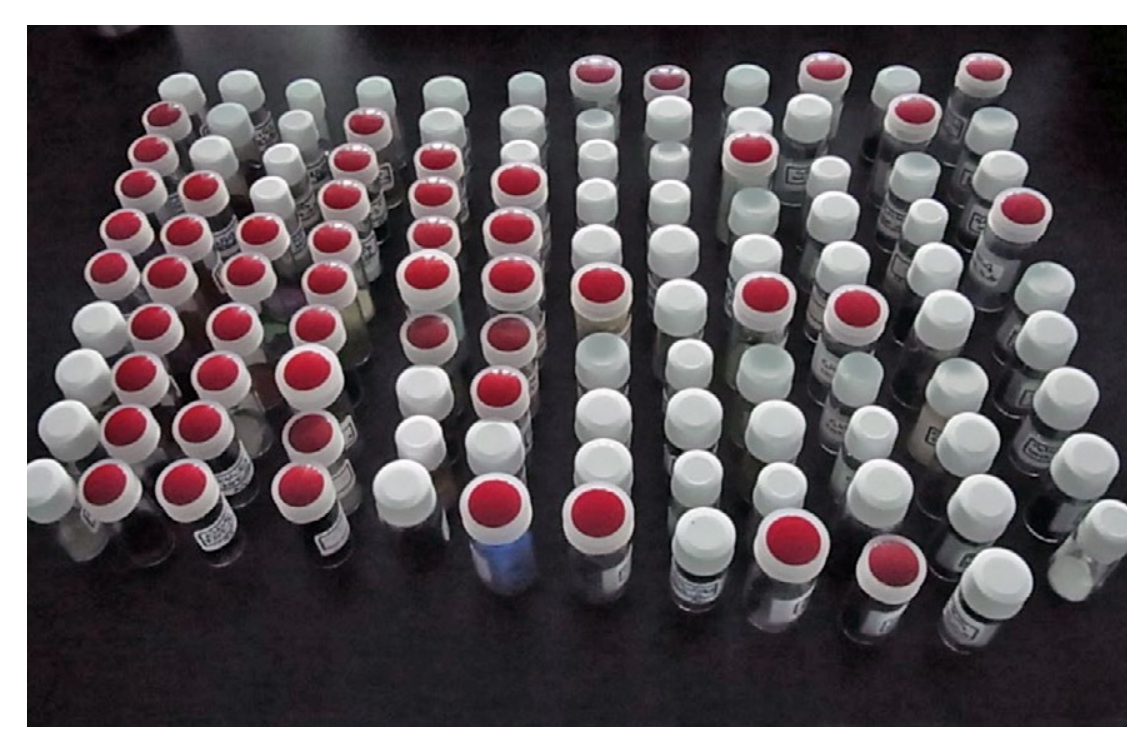

Snapshot of the various novel
the course of this study.

Dr Masese's materials are helping to herald in the post-lithium-ion battery age. exist as charged ions. Conventional electrolytes contain organic solvents, rendering them flammable. They also tend to decompose at high voltages and temperatures. lonic liquids do not contain flammable organic solvents. Therefore, they are safe and stable at high voltages and high temperatur which are beyond the capacity of conventional electrolytes.

Solid electrolytes are also highly desirable to combat some of the safety concerns about rechargeable ion batteries. Liquid electrolytes can leak if the battery is damaged and are also highly flammable, which is the origin of many of the highprofile stories about mobile phone battery fires. The problem with solid electrolytes is that, although they are better from a safety perspective, they

CRYSTAL STRUCTURE

P2-Type layered structure
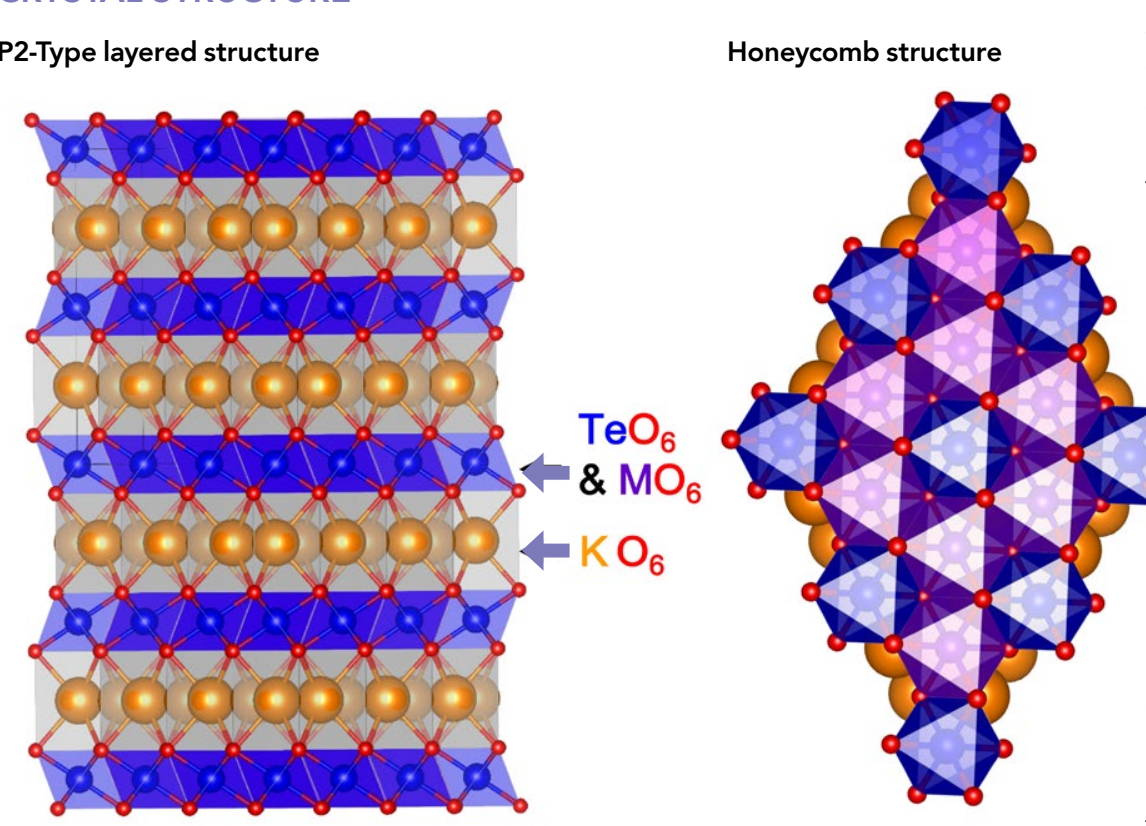

SIDE VIEW

TOP VIEW

The figure on
from above.

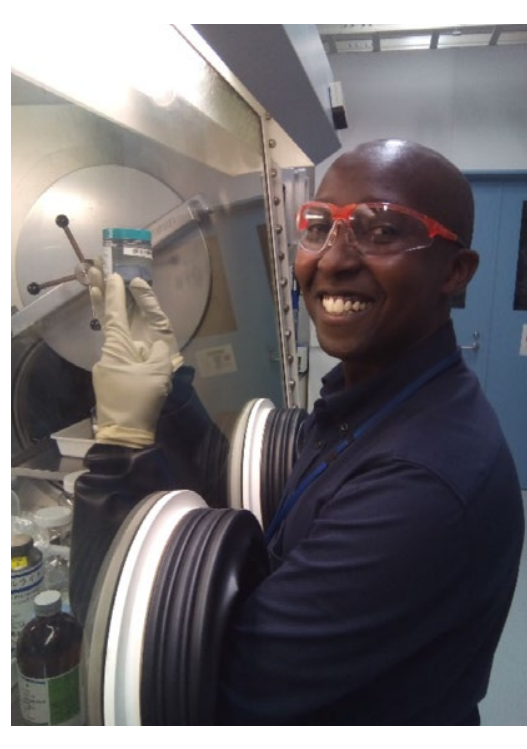

Dr Masese at work in the lab.

do not show the same efficiency as their iquid counterparts, but Dr Masese has demonstrated that the tellurate-based materials do show high conductivity for the potassium ions and may well play an important role in the development of solid electrolytes for potassium-ion batteries of the future.

LIGHTENING THE LOAD One of the key advantages of the potassium-ion batteries that Dr Masese is helping to realise is their very high voltage capabilities, that he has demonstrated through the performance of the electrode material. High-voltage supplies reduce the need for so many cells within a battery pack. This then entails small volume, cost and weight battery packs, something that is essentia instance, electric vehicles. The $85 \mathrm{kWh}$

battery pack in the Tesla Model S weigh in excess of an enomous $500 \mathrm{~kg}$ and accounts for nearly a quarter of the total weight of the car. Although 40 litres of petrol weighs aroun $30 \mathrm{~kg}$ and there is the weight of the fuel tank and fuelling system to consider, finding a solution for the heavy weight and bulky sizes of electrical batteries would dramatically increase the feasibility of electric vehicles and significantly increase their efficiency.

Dr Masese's materials are helping Therald in the post-lithium-ion battey age and demonstrate a significant based technologies.

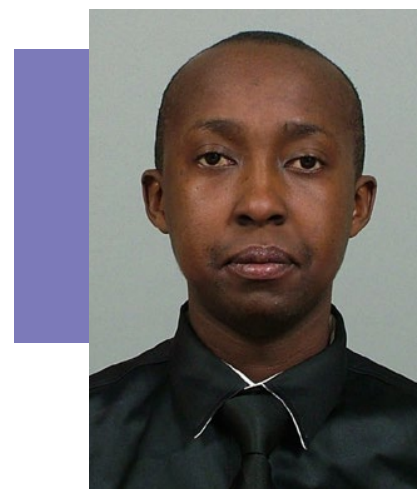

\section{Behind the Research}

Dr Titus Masese

E: titus.masese@aist.go.jp T: $\pm 81-72-751-9224 \quad$ W: www.aist.go.jp/index en.html

W: https://unit.aist.go.jp/riecen/index en.html_W: $\mathbf{h t t p s : / / w w w . y o u t u b e . c o m / w a t c h ? v = H O | 8 A J \times C A l w}$

Research Objectives

Working with the Advanced Battery Research Group AIST, Dr Masese's research explores high performance text-generation rechargeable battery systems.

\section{Detail}

DrTitus Masese

National Institute of Advanced Industrial Science and Technology (AIST)

8-31 Midorigaoka, keda-shi, Osaka, Japan

PO Box 563-8577 Kansai Center

Bio

Titus Masese hails from Kenya. He won a Japanese government scholarship after emerging amongst the top 列 his BEng, MSc and PhD degrees from Kyoto University (BEng supervisor: Prof Haruyuki Inui; PhD supervisor: Prof Yoshiharu Uchimoto). He is currently a researcher at AIST.

Funding

(n)

and Technogy (AST)

Collaborators

DryoichiYamaguchi

DrMinamiKato

- Dr Satoshi Uchida

- Dr Toyoki Okumura

- Dr Keigo Kubota

- Dr Hiroshi Senoh

- Prof Zhen-Dong Huang (Nanjing University)

- Prof Yuki Orikasa (Ritsumeikan University)

- Dr Hajime Matsumoto

- Prof Martin Månsson (KTH Royal Institute of Technology) Many thanks to Ms Kumi Shiokawa for the relentless technical assistance

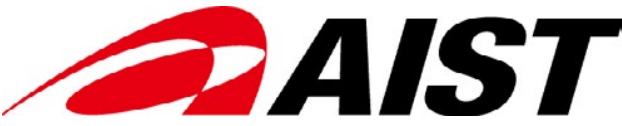

\section{References}

Masese, T. et al., (2018), Rechargeable potassium-ion batteries with honeycomb-layered tellurates as high voltage cathodes and fast potassium-ion conductors,

Masese, T. et al., (2019). A high voltage honeycomb layered cathode framework for rechargeable potassiu Communications, 55,985

Kato, M. et al., (2019), Organic positive-electrode material utilizing both an anion and cation: a benzoquinonetetrathiafulvalene triad molecule, Q-TTF-Q, for rechargeable $\mathrm{Li}, \mathrm{Na}$, and $\mathrm{K}$ batteries, New Journal of Chemistry, 43, 1626.

\section{Personal Response}

What are the next steps in your work to turn these honeycomb materials into a part of battery

II From a fundamental point of view, we have shown that high voltages are attainable with the honeycomblayered tellurate materials. Although tellurium has technological perspectives, its use may render these onging work is the design of tellurium-free related materials also demonstrating high voltages. While we note that there are other challenges that should ultimately be solved for the nascent potassium-ion technology to reach the market, the insights garnered in this study as well as other studies reported so far forward to designing a high-voltage battery prototype. We believe that by reaching out to the wide-spanning scientific community, we can bring the potassium-ion technology closer to reality. 